\title{
Chronic and acute effects of red wine versus red muscadine grape juice on body composition, blood lipids, vascular performance, inflammation, and antioxidant capacity in overweight adults
}

This article was published in the following Dove Press journal:

International Journal of Wine Research

Lisa S McAnulty

Scott R Collier ${ }^{2}$

Martin L Hubner ${ }^{2}$

Gregory Anoufriev²

Steven R McAnulty ${ }^{2}$

'Department of Nutrition and Health Care Management, ${ }^{2}$ Department of Health and Exercise Science, Appalachian State University, Boone, NC 28608, USA
Correspondence: Steven R McAnulty Department of Health and Exercise Science, Leon Levine Hall, Appalachian State University, Boone, NC 28608, USA Tel +| $828262715 \mid$

Fax +I 8282623138

Email mcanltysr@appstate.edu
Purpose: Red wine may benefit health due to the presence of polyphenolic compounds. This research investigated effects of wine (W) versus grape juice $(\mathrm{J})$ on body composition, blood lipids (cholesterol, HDL, LDL, and triglycerides [TG]), vascular responses (augmentation index $[\mathrm{AIx}]$ and central pulse wave velocity [cPWV]), inflammation (C-reactive protein $[\mathrm{CRP}]$ ), and plasma antioxidant capacity (ferric-reducing ability of plasma [FRAP]) in sedentary individuals.

Methods: In a randomized crossover design, 19 participants consumed $300 \mathrm{~mL}$ of $\mathrm{W}$ or $\mathrm{J}$ for two weeks and then acutely in the lab. Blood was drawn at baseline, post two weeks, and within $1 \mathrm{hr}$ after consuming treatment. Repeated measures ANOVA with 2 (treatment) $\times 3$ (time) was used for FRAP, AIx, and cPWV and 2 (treatment) $\times 2$ (time) for blood lipids and CRP. A paired $t$-test was used to compare differences in diet and weight change.

Results: Acute, but not chronic, consumption of wine significantly increased FRAP (treatment $P=0.028$ ) and significantly decreased AIx (treatment $P=0.038$ ) while juice exhibited no effects. An overall treatment effect existed for TG $(P=0.028)$ in wine only. Cholesterol, LDL, HDL, and CRP were not affected in either group.

Conclusion: Acute, but not chronic, consumption of wine significantly increased antioxidant capacity and resulted in beneficial changes to the vasculature as determined by AIx.

Keywords: antioxidants, antioxidant capacity, lipids, cardiovascular function, inflammation, wine

\section{Introduction}

Red wine has been associated with multiple health effects such as decreased risk for total mortality, coronary artery disease (CAD), congestive heart failure, and stroke.${ }^{1-6}$ Wine can have a varying complex composition which is affected by red versus white and the variety of grapes. ${ }^{7}$ Alcohol and multiple phenolic compounds, primarily the stilbene resveratrol and anthocyanins, have been suggested to produce the benefits ascribed to wine. ${ }^{8-10}$

The phenolic compounds present in red wine are mainly responsible for the total antioxidant capacity of red wines. Specifically, glycosylated and methoxylic anthocyanins such as malvidin-3-glucoside are associated with antioxidant effects. ${ }^{11}$ Wine may also increase plasma antioxidant capacity, as exhibited by increases in the ferricreducing ability of plasma (FRAP), and may subsequently mitigate the effects of oxidative stress. ${ }^{6,12}$ Fernandez-Pachon et al $^{13}$ investigated plasma antioxidant capacity 
in eight healthy human volunteers after wine intake. FRAP values were determined before ingestion of $300 \mathrm{~mL}$ of red wine (baseline) and 30, 55, and 120 mins following consumption. The maximum average increase in FRAP values was reached at 55 mins. As previously stated, wine may increase antioxidant capacity in humans, but the actual benefits of this increase have not been clearly elucidated.

Oxidative stress and inflammation are increased in aging and obese animal and human models. Specifically, obesity is associated with increased free radical production as well as increased inflammation markers, such as Creactive protein (CRP), which is associated with an increased risk of cardiovascular disease (CVD). ${ }^{14,15}$ An established method to monitor changes in risk for CVD is to monitor vascular changes such as the stiffness of the arteries. This can be effectively evaluated by using augmentation index (AIx). AIx is an indicator of arterial wave reflection at the aorta measured as percent reflected. A greater percentage indicates greater overall reflection of the pulse wave which is indicative of greater CVD risk and poorer vascular health, whereas a lower reflected percentage is indicative of better vascular health. ${ }^{16}$ Mahmud et $\mathrm{al}^{17}$ found that, when ingested acutely, red wine containing alcohol but not dealcoholized wine reduced blood pressure, central pulse wave velocity (cPWV), and AIx.

Muscadine grapes and wine are indigenous to the Southeastern United States making them a popular choice for local consumers. Consumption of muscadine grape and wine phytochemicals in the diet may help to prevent obesity-related metabolic complications. ${ }^{18}$ Most studies have been conducted with common varietals such as Cabernet Sauvignon or Merlot, and very few have investigated muscadine-based wines. ${ }^{19}$ Although epidemiological evidence suggests that moderate consumption of red wine is associated with a decrease in CVD, ${ }^{6,20,21}$ the comprehensive effects of red wine, specifically muscadine wine, on vascular changes such as AIx and cPWV have not been examined in connection with changes in body composition, blood lipid profiles, inflammatory state, and antioxidant capacity.

Given that obesity and a sedentary lifestyle exert detrimental effects such as inflammation, oxidative stress, and reduced vascular function, the purpose of this study was to examine the effect of daily and acute muscadine wine versus muscadine grape juice on body weight, body composition, blood lipids, vascular function, inflammation, and antioxidant capacity in a sedentary and overweight-obese population. We sought to improve and expand upon previous work by evaluating both chronic and acute changes in these parameters as they relate to each other.

\section{Materials and methods \\ Participants}

Nineteen volunteers who were overweight or obese (body mass index [BMI] >24.9), at least 40 years old, non-smoking, sedentary, and not on prescription medications were recruited, screened, and signed a written informed consent document. All participants were infrequent consumers of alcohol (ie consuming $\leq$ three alcoholic drinks per every two weeks). All participants were screened using a standardized Health and Medical History Screening Form. Furthermore, to determine that our population was sedentary, we utilized a 10 point scale with 10 being extremely active and 1 being completely sedentary. We divided the scale into thirds and identified anyone scoring less than 3.0 as being sedentary. Participants were randomized to a wine (W) group or grape juice (J) group and then crossed over into the opposing group, following a two-week washout period, where the identical protocol was repeated.

\section{General study design}

Participants were instructed to stop consuming fruits and fruit juices three days before each visit to the lab for testing. Use of medications and consumption of alcohol, other than wine provided, were also prohibited during the study. Further, herbal medicines or large-dose vitamin/ mineral supplements (above $100 \%$ of recommended dietary allowances) were not allowed during the study period, and participants were encouraged to maintain usual dietary patterns during the study. Participants were also instructed to be fasted for at least $8 \mathrm{hrs}$ prior to reporting to the lab.

All participants visited the Human Performance Laboratory four times throughout the study. At orientation (Visit 1), health history questionnaires and consent forms were obtained, along with height, weight, and body composition. Body composition was assessed by bioelectrical impedance. Baseline blood and vascular measures were also obtained. Participants were instructed on adherence to dietary restrictions which included not consuming other alcoholic beverages, grapes, or grape products during the study. Participants were given instruction on recording a three-day diet record for two weekdays and one weekend day during the treatment weeks. After participants were randomized to their respective treatments, wine $(\mathrm{W})$ or grape juice $(\mathrm{J})$ was given to each participant. At home, the $\mathrm{W}$ group consumed two $150 \mathrm{~mL}$ servings of wine per day for two weeks, and the $\mathrm{J}$ group 
consumed grape juice with the same number of servings, serving size, and duration as the $\mathrm{W}$ group. Compliance was assessed by having participants return all bottles to the laboratory on the same day in which testing occurred.

On the second visit, two weeks after baseline, participants returned 3-day diet records, and weight, percent body fat, blood, and vascular measures were again obtained. To assess acute effects, each participant then consumed a single $300 \mathrm{~mL}$ serving of either red wine or grape juice, respective to group assignment. Another blood draw was taken precisely $1 \mathrm{hr}$ after consumption. To ensure safety, participants remained in the lab for $1 \mathrm{hr}$ after the acute consumption and were given a small meal before leaving. Participants then had a washout period of two weeks during which they refrained from drinking wine or grape juice.

Upon returning for the third visit, weight, percent body fat, blood, and vascular measures were obtained. The protocol was repeated with participants crossing over into the opposing treatment (wine treatment switched to grape juice treatment and vice-versa) for two weeks. The fourth lab visit protocol was the same as described in visit two. Participants also provided another set of three-day diet records. Figure S1 provides a detailed study flow diagram.

\section{Wine and grape juice samples}

Hatteras Red ${ }^{\mathrm{TM}}$ wine (Duplin Winery Rose Hill, NC) contains $12 \%$ alcohol by volume and is made from muscadine grapes. Muscadine grape juice (100\% grape juice) was provided by D'Vine Foods (Elizabethtown, NC) and used as the placebo. Heat and direct sunlight were avoided when storing the red wine and grape juice. Once opened, the wine and juice were stored sealed in a refrigerator.

\section{Blood}

Blood was obtained by venipuncture of the antecubital vein, with participants in the seated position. One $5 \mathrm{~mL}$ EDTA tube of blood was drawn for antioxidant assessment (FRAP) and centrifuged at 3,000 rpm for $10 \mathrm{mins}$ at $3{ }^{\circ} \mathrm{C}$. Plasma collected for analysis of FRAP was aliquoted into snap-top tubes $(0.5 \mathrm{~mL})$, immediately frozen in liquid nitrogen, and stored at $-80{ }^{\circ} \mathrm{C}$ for further analysis. One 5 $\mathrm{mL}$ serum-separator tube (SST) of blood was collected for assessing CRP, cholesterol, HDL, LDL, and TG. The SST tubes sat for 20 mins to allow blood to clot.

\section{Ferric-reducing ability of plasma}

The FRAP test was used to analyze all blood samples for assessment of antioxidant capacity according to the methodology of Benzie and Strain. ${ }^{22}$ Reducing refers to when a reactive species, or oxidant, is reduced by the reductant, or antioxidant. This redox reaction causes one species to be reduced and the other to be oxidized by gaining and losing electrons, respectively. In FRAP, a ferric $\left(\mathrm{Fe}^{\mathrm{III}}\right)$ compound is reduced to the ferrous $\left(\mathrm{Fe}^{\mathrm{II}}\right)$ form. This reduction will take place when a reductant, or antioxidant, is present in the substance. In the analysis, in vitro, this reduction results in the appearance of a blue chromogen. Therefore, the intensity of the blue color correlates to the amount of water-soluble antioxidants present in the sample. ${ }^{22}$ For this study, a working FRAP reagent was composed of acetate buffer, TPTZ solution, and an iron chloride plus water solution. Standards and samples were analyzed by adding the FRAP reagent and measuring absorbance. The standard was an ascorbate solution made of ascorbic acid and distilled water, and the samples were the plasma collected from each participant. Each sample and standard were measured in triplicate.

\section{Analysis of the effects, in vitro, of alcohol on antioxidant capacity}

The FRAP assay was utilized to examine the effect of the addition of exogenous ethanol to the grape juice that was used in this study. In this experiment, absolute ethanol was added to the grape juice to make a $12 \%$ solution, in order to equal the alcohol concentration of the wine, and FRAP was examined in triplicate. Also, in order to examine the ethanol component alone, an experiment was conducted in which a solution of deionized water (DI) and ethanol $(12 \%)$ was prepared and FRAP examined in triplicate.

\section{Blood lipids and CRP}

SSTs were delivered to the biochemistry laboratory at Charles A. Cannon Jr., Memorial Hospital located in Linville, North Carolina, for assessment of CRP and blood lipids using automated procedures.

\section{Augmentation index and central pulse wave velocity}

A SphygmoCor (AtCor, New South Wales, Australia) was used to measure AIx and cPWV. Participants were measured at the beginning of the study (baseline), post-chronic consumption, and post-acute consumption for both wine and grape juice groups. All measurements were conducted in accordance with guidelines set forth by the Clinical Application of Arterial Stiffness, Task Force III. Fifteen separate waveforms were taken and averaged for AIx and cPWV. A SphygmoCor was used to obtain the pulse wave between 
(1) the left common carotid artery and left femoral artery and

(2) between the left common carotid and left dorsalis pedis artery. Distance from the carotid sampling site to the midpoint of the manubrium sterni, manubrium sternum to femoral artery, and carotid to the mid-point of the manubrium sterni, manubr ium sternum to dorsalis pedis was measured between these points as straight lines with a tape measure. The distance from the carotid artery to the manubrium sterni was subtracted from the manubrium to femoral artery distance.

Central pulse wave velocity was determined from the foot-to-foot flow wave velocity. The foot of the pressure wave was identified visually as the point of systolic upstroke. Central pulse wave velocity was then calculated from the distances between measurement points and the measured time delay (Dt) between proximal and distal foot waveforms as follows: $\mathrm{Cpwv}=\mathrm{D} / \mathrm{Dt}(\mathrm{m} / \mathrm{s})$ where $\mathrm{D}$ is distance in meters and $\mathrm{Dt}$ is the time interval in seconds. Values attained from carotid to femora 1 artery were taken as an index of central compliance, while values attained from the carotid and radial artery along with the measurement from the femoral to dorsalis pedis were taken as an index of peripheral compliance. The cPWV measurement used the pressure sensors placed precisely over the carotid artery in the neck and the femoral artery in the groin to measure the speed with which pulse waves travel down the aorta. AIx was determined by the change in pressure between the first and second peaks divided by the pulse pressure $(\mathrm{AIx}=$ change $\mathrm{P} / \mathrm{PP})$.

\section{Polyphenolic content of wine and grape juice}

HPLC was utilized via a Dionex U3000 HPLC System (Dionex, Sunnyvale, CA, USA). Samples of red muscadine wine and red muscadine grape juice were analyzed for polyphenol content using previously defined methods. ${ }^{23}$

\section{Data analysis}

Statistical tests were performed using SPSS 16.0 for Windows (SPSS Inc., Chicago, IL, USA). A randomized crossover design utilized a 2 (treatment) $\times 3$ (time) repeated measures ANOVA (FRAP and AIx) and a 2 (treatment) $\times 2$ (time) repeated measures ANOVA for total cholesterol, TG, LDL, HDL, and CRP. A paired $t$-test was used to compare differences in diet and anthropometrics. All values were expressed as mean $\pm \mathrm{SD}$, except AIx and $\mathrm{cPWV}$ which were expressed as mean \pm SEM. An a priori $P$ value $\leq 0.05$ was considered statistically significant. The three- day diet records were analyzed using Food Processor SQL software (ESHA Research, Salem, OR, USA). Any data outside of \pm 2.5 SD were considered as outliers and were excluded from statistical analysis.

\section{Results}

Twenty-six original participants were recruited with 19 completing all stages of the study. Participant characteristics are presented in Table 1. The mean BMI was 32.1 \pm 3.8 . Mean body mass $(\mathrm{kg})$ was $90.9 \pm 15.1$. Interestingly, males and females did not statistically differ in height and weight. No differences existed between groups with respect to energy, macronutrients, or micronutrients (data not shown). Participants ranged in age from 40 to 58, with six being overweight and 13 being obese. The pre-medical screen found that all participants consumed $\leq 3$ alcoholic drinks per every two week period. Table 2 indicates that the weight change and total fat mass percentage, respectively, in the wine and grape juice groups were not significantly different after treatments. Table 3 indicates that CRP (Time $P=0.194$, Interaction $P=0.894$ ), blood cholesterol (Time $P=0.735$, Interaction $P=0.932$ ), HDL (Time $P=0.440$, Interaction $P=0.373$ ), and LDL (Time $P=0.101$, Interaction $P=0.418$ ) were not significantly different. Data indicate that, in all cases, there were no significant effects of time or interaction between these variables. However, triglycerides were significantly increased by wine consumption (treatment effect $P=$ 0.028). HPLC analysis of the wine and grape juice revealed that the wine was higher in quercetin and $\mathrm{p}$ coumaric acid compared to juice. Juice was significantly higher in gallic acid and epicatechin compared to wine, but no other differences existed. The polyphenolic contents of the wine and grape juice are shown in Table 4.

AIx, as shown in Figure 1, was significantly reduced acutely after consumption of wine $(P \leq 0.01)$ and was unchanged following consumption of grape juice. Central pulse wave velocity (Figure 2) was not significantly

Table I Baseline characteristics of participants

\begin{tabular}{|l|l|}
\hline Gender & $\mathbf{8}$ males, I I females \\
\hline Age $(\mathrm{yrs})$ & $48.7 \pm 5.2$ \\
Height $(\mathrm{m})$ & $1.6 \pm 0.09$ \\
Body mass $(\mathrm{kg})$ & $90.9 \pm 15.1$ \\
BMI $\left(\mathrm{kg} / \mathrm{m}^{2}\right)$ & $32.1 \pm 3.8$ \\
Baseline alcohol consumption & $\leq \mathbf{3}$ drinks/2 weeks \\
\hline
\end{tabular}

Notes: Values are mean $\pm S D$. $(N=19)$. 
Table 2 Body composition after two weeks daily consumption of red wine and grape juice

\begin{tabular}{|l|l|l|l|}
\hline & Wine & Juice & P value \\
\hline Baseline wt (kg) & $90.9 \pm 15$ & $91.6 \pm 15.3$ & 0.887 \\
Post two weeks wt (kg) & $91.5 \pm 15.2$ & $91.8 \pm 15.4$ & 0.952 \\
Baseline body fat (\%) & $36.7 \pm 6.8$ & $36.5 \pm 6.7$ & 0.927 \\
Post two weeks body fat (\%) & $36.3 \pm 6.3$ & $36.8 \pm 6.3$ & 0.808 \\
\hline
\end{tabular}

Notes: Values are mean \pm SD. Wine to juice cross comparisons for each variable were not significantly different. Furthermore, baseline to post two weeks comparisons within wine and juice groups were also not significantly different $(\mathrm{N}=19)$.

Table 3 CRP and lipids after two weeks daily consumption of red wine and grape juice

\begin{tabular}{|c|c|c|c|}
\hline & Wine & Grape juice & $P$ value (treatment) \\
\hline \multicolumn{4}{|c|}{ C-reactive protein (mg/L) } \\
\hline Baseline & $3.7 \pm 2.6$ & $3.8 \pm 2.6$ & 0.774 \\
\hline Post two weeks & $4.14 \pm 2.9$ & $4.3 \pm 3.2$ & \\
\hline \multicolumn{4}{|l|}{ Cholesterol (mg/dL) } \\
\hline Baseline & $216.5 \pm 34.7$ & $211.1 \pm 36.4$ & 0.219 \\
\hline Post two weeks & $215.3 \pm 32.4$ & $209.3 \pm 35.2$ & \\
\hline \multicolumn{4}{|l|}{ HDL (mg/dL) } \\
\hline Baseline & $47.3 \pm 14.1$ & $47.5 \pm 14.2$ & 0.572 \\
\hline Post two weeks & $47.0 \pm 15.6$ & $45.6 \pm 13.7$ & \\
\hline \multicolumn{4}{|l|}{ LDL (mg/dL) } \\
\hline Baseline & $141.8 \pm 28.8$ & $139.3 \pm 29.9$ & 0.906 \\
\hline Post two weeks & $133.0 \pm 24.7$ & $136.7 \pm 30.3$ & \\
\hline \multicolumn{4}{|l|}{ Triglycerides (mg/dL) } \\
\hline Baseline & $135.6 \pm 32.8$ & $121.1 \pm 42.7$ & 0.028 \\
\hline Post two weeks & a $147.9 \pm 45.7$ & $121.0 \pm 31.9$ & \\
\hline
\end{tabular}

Notes: ${ }^{a}$ Significantly different from the baseline value. Values are mean $\pm S D(N=19)$.

Table 4 Polyphenolic content of wine and grape juice

\begin{tabular}{|l|l|l|l|}
\hline Polyphenol $(\boldsymbol{\mu g} / \mathbf{m L})$ & Wine & Juice & P value \\
\hline Gallic & $30 \pm 0.0$ & $36.5 \pm 1.0$ & $\leq 0.001$ \\
Catechin & $7.5 \pm 1.0$ & $8.7 \pm 1.0$ & $\mathrm{NS}$ \\
Caffeic & $3.0 \pm 0.7$ & $3.8 \pm 0.7$ & $\mathrm{NS}$ \\
Epicatechin & $6.5 \pm 1.0$ & $11.1 \pm 0.8$ & $\leq 0.001$ \\
-Coumaric & $1.18 \pm 0.5$ & $0.4 \pm 0.9$ & $\leq 0.001$ \\
Quercetin & $2.36 \pm 0.8$ & $0.9 \pm 0.4$ & $\leq 0.001$ \\
\hline
\end{tabular}

Note: Values are mean $\pm S D(N=6)$.

Abbreviation: NS, not significantly different.

affected by either treatment. Plasma antioxidant capacity, as measured by FRAP, significantly increased acutely with wine only ( $\mathrm{P} \leq 0.001)$ (Figure 3$)$. The in vitro analysis of the effects of ethanol being added to grape juice and of ethanol alone resulted in no change in FRAP-values (data not shown).

\section{Discussion}

Consumption of red wine is probably best known for exerting cardioprotective benefits. Several studies report improved endothelial function as a result of red wine and/ or grape juice consumption. ${ }^{20,24,25}$ This improved endothelial function was primarily demonstrated through enhanced 


\section{Augmentation index (Alx)}

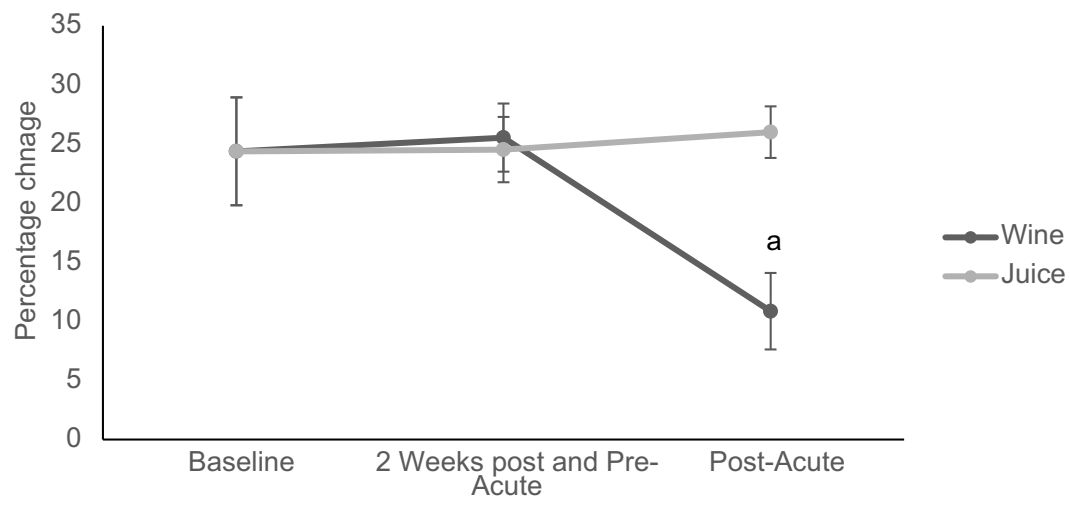

Figure I Changes in augmentation index (Alx). Augmentation index significantly declined after the two weeks post- and pre-acute wine consumption compared to juice consumption. Treatment $(P=0.015)$ eta ${ }^{2}=0.284$, Time $(P=0.056)$ eta ${ }^{2}=0.510$, Treatment-Time Interaction $(P=0.291)$ eta ${ }^{2}=0.049$. ${ }^{a}$ Baseline wine versus post-acute $(P \leq$ $0.001)$. Values are mean $\pm \operatorname{SEM}(\mathrm{N}=19)$.

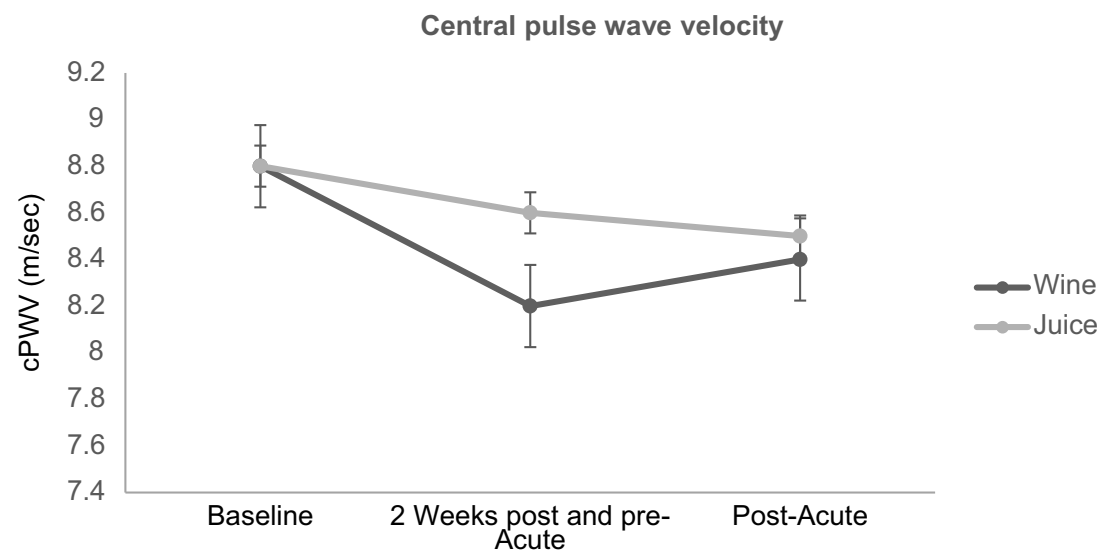

Figure 2 Changes in pulse wave velocity $(\mathrm{cPWV})$. Central pulse wave velocity was unchanged after both wine and juice consumption. Treatment $(P=0.422)$ eta ${ }^{2}=0.054$, Time $(P=0.712)$ eta ${ }^{2}=0.028$, Treatment-Time Interaction $(P=0.271)$ eta ${ }^{2}=0.103$. Values are mean $\pm \mathrm{SEM}(\mathrm{N}=19)$.

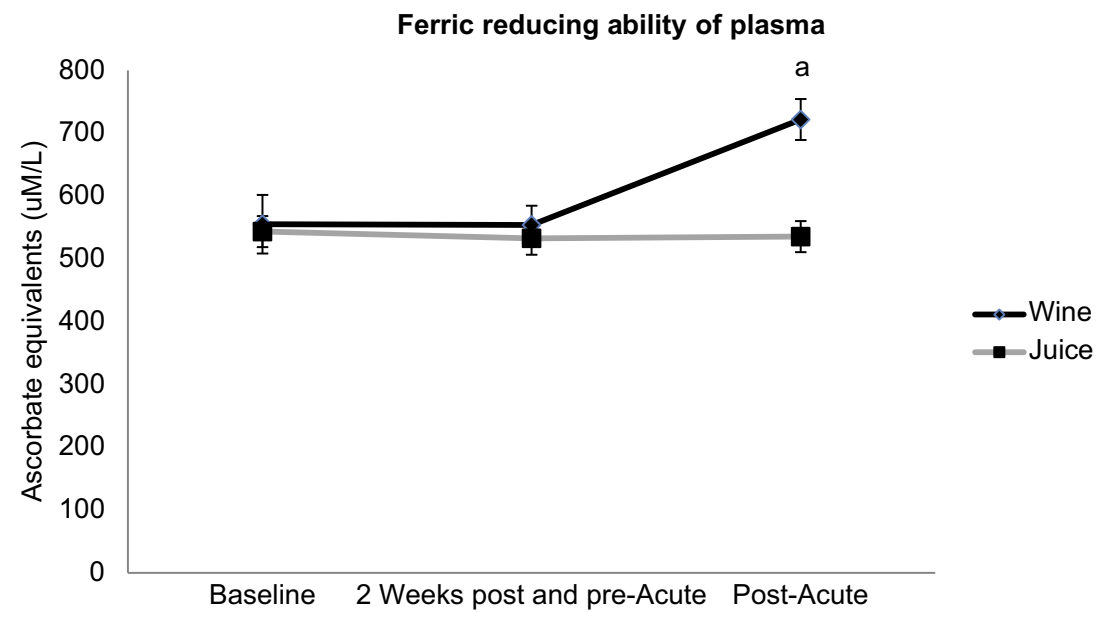

Figure 3 Changes in ferric-reducing ability of plasma (FRAP). ${ }^{a}$ Ferric reducing ability of plasma significantly increased after acute wine consumption but not chronic consumption $(P=0.00 \mathrm{I})$. Treatment $(P=0.028)$ eta ${ }^{2}=0.253$, Time $(P=0.160)$ eta ${ }^{2}=0.11 \mathrm{I}$, Treatment-Time Interaction $(P=0.125)$ eta ${ }^{2}=0.130$. Values are mean $\pm \mathrm{SEM}$ $(\mathrm{N}=19)$. 
eNOS function. ${ }^{26}$ Therefore, there is good evidence to suggest that red wine increases eNOS expression and subsequent endothelial NO release, thereby affecting vessel elasticity. ${ }^{3,26,27}$ In support of this concept, the main findings of this study reveal that acute red wine consumption, but not grape juice, resulted in an increase in FRAP associated with a decrease in AIx but exerted no changes to blood lipids or CRP. Increases in AIx correlate with increases of arterial stiffness induced by aging, atherosclerosis, or arterial hypertension and have a prognostic value for cardiovascular events. Our findings are in accordance with studies which cite evidence to support that red wine has cardioprotective benefits. ${ }^{6,20,21}$ These vascular benefits are typically attributed to the polyphenol content within the red wine. Grapes and wines can exhibit very high antioxidant values, in vitro, allowing the assumption that these foods can serve as very potent antioxidants although less is known about effects in vivo. $^{12,28,29}$

Additionally, several studies have shown that the ethanol content combined with the polyphenol content of red wine helps improve and/or maintain endothelial function. ${ }^{20,30-32}$ In support of this concept, we found that only the acute red wine consumption resulted in a significant increase in FRAP and a significant decrease in AIx. Furthermore, it was concluded from our experiments, in vitro, that the combined effect of the ethanol and grape polyphenols induces a rise in FRAP values, but ethanol alone does not elicit any effect on antioxidant capacity as determined by FRAP. Kiviniemi et $\mathrm{al}^{24}$ found no effects on antioxidant capacity after acute consumption of de-alcoholized red wine. In agreement with our findings, other investigators ${ }^{33,34}$ have demonstrated that FRAP, after acute consumption of red wine and measured at various time intervals, shows significantly elevated values when compared to pre-consumption values.

AIx and cPWV represent measurements of aortic elasticity and can be useful in determining an individual's risk for developing CVD. Following acute wine consumption, we observed an increase in FRAP associated with a concurrent decline in AIx and no change in cPWV. Our results partially agree with those of Karatzi et $\mathrm{al}^{3}$ who investigated the effects of red wine and de-alcoholized red wine on aortic pressures and arterial stiffness in patients with $\mathrm{CAD}$. Both regular and de-alcoholized red wine caused a significant decrease in AIx, whereas no significant change was induced in cPWV. The authors concluded that both types of red wine provoked favorable acute effects on wave reflections, but the largest effect was attributed mainly to red wine antioxidant substances, although antioxidant capacity was not assessed. This partially supports our observation that acute ingestion of wine decreases AIx but not cPWV.

There are several limitations in this study. One limitation is the use of overweight and obese participants which limits the generalizability of findings. Another is the lack of an absolute placebo, such as drinking water or white wine, since both groups consumed phenolic antioxidants. This resulted in the difference between the treatments being the presence of ethanol and a larger antioxidant capacity in the wine; however, juice was chosen as a placebo for a few reasons determined in our pre-project analysis: 1) HPLC analysis of the wine and juice found the wine to be significantly higher in quercetin and p-coumaric, 2) addition of different concentrations of alcohol to deionized water did not register any FRAP value. Therefore, it was predicted that any differences between wine and juice would be from differences in phenolic content. A final limitation of the study was the small sample size that may have hampered the robustness of the findings for some variables.

\section{Conclusions}

We observed that red wine, but not grape juice, caused a significant increase in FRAP and a significant decrease in AIx following acute consumption. We did not observe significant chronic changes in body composition, cholesterol, HDL, LDL, CRP, AIx, cPWV, or FRAP following daily consumption of muscadine wine or grape juice for two weeks in middle-aged, overweight, or obese individuals. An overall treatment effect was observed for triglycerides which increased after two weeks of wine, but subsequent post-hoc testing revealed no significant changes between groups. We conclude the presence of ethanol might be necessary to enhance the absorption of the phenolic compounds in wine or that the difference in phenolic content between wine and grape juice is important to elicit beneficial effects. We further conclude that the health benefits associated with wine consumption are most likely limited to acute effects or that longer periods of wine consumption, beyond two weeks, are needed to exhibit these benefits.

\section{Abbreviation list}

FRAP, Ferric reducing ability of plasma; CVD, Cardiovascular disease; CRP, C- reactive protein; cPWV, Central pulse wave velocity; AIx, augmentation index; BMI, body mass index; HDL, high density lipoprotein; LDL, low density lipoprotein; TG, triglyceride; HPLC, 
High Pressure Liquid Chromatography; EDTA, Ethylene diamine tetraacetic acid; SST, serum separator tube; IRB, Institutional Review Board.

\section{Institutional Review}

All procedures regarding study design were reviewed and approved by the Institutional Review Board (IRB) at Appalachian State University, Boone, North Carolina.

\section{Acknowledgments}

The authors wish to acknowledge the following organization and businesses for their support in this project:

\section{University Research Council - Appalachian State University \\ 2. Duplin Winery - Rose Hill, North Carolina - provided wine \\ 3. D'Vine Foods - Elizabethtown, NC - provided grape juice.}

\section{Disclosure}

The authors report no conflicts of interest in this work.

\section{References}

1. Carbonneau MA, Leger CL, Monnier L, et al. Supplementation with wine phenolic compounds increases the antioxidant capacity of plasma and vitamin $\mathrm{E}$ of low-density lipoprotein without changing the lipoprotein $\mathrm{Cu}(2+)$-oxidizability: possible explanation by phenolic location. Eur J Clin Nutr. 1997;51(10):682-690.

2. Leighton F, Cuevas A, Guasch V, et al. Plasma polyphenols and antioxidants, oxidative DNA damage and endothelial function in a diet and wine intervention study in humans. Drugs Exp Clin Res. 1999;25(2-3):133-141.

3. Karatzi KN, Papamichael CM, Karatzis EN, et al. Red wine acutely induces favorable effects on wave reflections and central pressures in coronary artery disease patients. Am J Hypertens. 2005;18(9 Pt 1):1161-1167. doi:10.1016/j.amjhyper.2005.03.744

4. Rifler JP, Lorcerie F, Durand P, et al. A moderate red wine intake improves blood lipid parameters and erythrocytes membrane fluidity in post myocardial infarct patients. Mol Nutr Food Res. 2012;56(2):345351. doi: $10.1002 / \mathrm{mnfr} .760$

5. Apostolidou C, Adamopoulos K, Lymperaki E, Iliadis S, Papapreponis P, Kourtidou-Papadeli C. Cardiovascular risk and benefits from antioxidant dietary intervention with red wine in asymptomatic hypercholesterolemics. Clin Nutr ESPEN. 2015;10(6):e224-e233. doi:10.1016/j.clnesp.2015.08.001

6. Golan R, Gepner Y, Shai I. Wine and health-new evidence. Eur J Clin Nutr. 2018. doi:10.1038/s41430-018-0309-5

7. Artero A, Artero A, Tarin JJ, Cano A. The impact of moderate wine consumption on health. Maturitas. 2015;80(1):3-13. doi:10.1016/j. maturitas.2014.09.007
8. Bhavnani BR, Cecutti A, Gerulath A, Woolever AC, Berco M. Comparison of the antioxidant effects of equine estrogens, red wine components, vitamin $\mathrm{E}$, and probucol on low-density lipoprotein oxidation in postmenopausal women. Menopause. 2018;25 (11):1214-1223. doi:10.1097/GME.0000000000001222

9. Cao H, Ou J, Chen L, et al. Dietary polyphenols and type 2 diabetes: human study and clinical trial. Crit Rev Food Sci Nutr;2018. 1-9. doi:10.1080/10408398.2018.1492900

10. Castello F, Costabile G, Bresciani L, et al. Bioavailability and pharmacokinetic profile of grape pomace phenolic compounds in humans. Arch Biochem Biophys. 2018;646:1-9. doi:10.1016/j. abb.2018.03.021

11. Rivero-Perez MD, Muniz P, Gonzalez-Sanjose ML. Contribution of anthocyanin fraction to the antioxidant properties of wine. Food Chem Toxicol. 2008;46(8):2815-2822. doi:10.1016/j.fct.2008.05.014

12. Dordevic NO, Todorovic N, Novakovic IT, et al. Antioxidant activity of selected polyphenolics in yeast cells: the case study of montenegrin merlot wine. Molecules. 2018;23(8). doi:10.3390/molecules23081971

13. Fernandez-Pachon MS, Villano D, Troncoso AM, Garcia-Parrilla MC. Antioxidant capacity of plasma after red wine intake in human volunteers. J Agric Food Chem. 2005;53(12):5024-5029. doi:10.1021/jf0501995

14. Pourshahidi LK. Vitamin D and obesity: current perspectives and future directions. Proc Nutr Soc. 2015;74(2):115-124. doi:10.1017/ S0029665114001578

15. Pourshahidi LK, Wallace JM, Mulhern MS, et al. Indices of adiposity as predictors of cardiometabolic risk and inflammation in young adults. J Hum Nutr Diet. 2016;29(1):26-37. doi:10.1111/jhn.12295

16. Beck DT, Martin JS, Nichols WW, Gurovich AN, Braith RW. Validity of a novel wristband tonometer for measuring central hemodynamics and augmentation index. Am J Hypertens. 2014;27(7):926931. doi:10.1093/ajh/hpt300

17. Mahmud A, Feely J. Divergent effect of acute and chronic alcohol on arterial stiffness. Am J Hypertens. 2002;15(3):240-243.

18. Gourineni V, Shay NF, Chung S, Sandhu AK, Gu L. Muscadine grape (Vitis rotundifolia) and wine phytochemicals prevented obesity-associated metabolic complications in C57BL/6J mice. J Agric Food Chem. 2012;60(31):7674-7681. doi:10.1021/jf3013663

19. Gonzalez-Centeno MR, Jourdes M, Femenia A, Simal S, Rossello C, Teissedre PL. Proanthocyanidin composition and antioxidant potential of the stem winemaking byproducts from 10 different grape varieties (Vitis vinifera L.). J Agric Food Chem. 2012;60 (48):11850-11858. doi:10.1021/jf303047k

20. Oak MH, Auger C, Belcastro E, Park SH, Lee HH, Schini-Kerth VB. Potential mechanisms underlying cardiovascular protection by polyphenols: role of the endothelium. Free Radic Biol Med. 2018;122:161-170. doi:10.1016/j.freeradbiomed.2018.03.018

21. O‘Keefe EL, DiNicolantonio JJ, O‘Keefe JH, Lavie CJ. Alcohol and CV health: Jekyll and Hyde J-curves. Prog Cardiovasc Dis. 2018;61 (1):68-75. doi:10.1016/j.pcad.2018.02.001

22. Benzie IF, Strain JJ. The ferric reducing ability of plasma (FRAP) as a measure of "antioxidant power": the FRAP assay. Anal Biochem. 1996;239(1):70-76. doi:10.1006/abio.1996.0292

23. Izquierdo-Llopart A, Saurina J. Characterization of sparkling wines according to polyphenolic profiles obtained by HPLC-UV/Vis and principal component analysis. Foods. 2019;8(1):22. doi:10.3390/ foods 8010022

24. Kiviniemi TO, Saraste A, Lehtimaki T, et al. Decreased endothelin-1 levels after acute consumption of red wine and de-alcoholized red wine. Atherosclerosis. 2010;211(1):283-286. doi:10.1016/j. atherosclerosis.2010.01.017 
25. Krnic M, Modun D, Budimir D, et al. Comparison of acute effects of red wine, beer and vodka against hyperoxia-induced oxidative stress and increase in arterial stiffness in healthy humans. Atherosclerosis. 2011;218(2):530-535. doi:10.1016/j.atherosclerosis.2011.07.004

26. Leighton F, Miranda-Rottmann S, Urquiaga I. A central role of eNOS in the protective effect of wine against metabolic syndrome. Cell Biochem Funct. 2006;24(4):291-298. doi:10.1002/cbf.1269

27. Lassaletta AD, Chu LM, Elmadhun NY, et al. Cardioprotective effects of red wine and vodka in a model of endothelial dysfunction J Surg Res. 2012;178(2):586-592. doi:10.1016/j.jss.2012.06.009

28. Garcia-Guzman JJ, Lopez-Iglesias D, Cubillana-Aguilera L, et al. Assessment of the polyphenol indices and antioxidant capacity for beers and wines using a tyrosinase-based biosensor prepared by sinusoidal current method. Sensors (Basel). 2018;19(1):66. doi:10.3390/s19010066

29. Pandeya A, Rayamajhi S, Pokhrel P, Giri B. Evaluation of secondary metabolites, antioxidant activity, and color parameters of Nepali wines. Food Sci Nutr. 2018;6(8):2252-2263. doi:10.1002/fsn3.794
30. Di Renzo L, Cioccoloni G, Sinibaldi Salimei P, Ceravolo I, De Lorenzo A, Gratteri S. Alcoholic beverage and meal choices for the prevention of noncommunicable diseases: a randomized nutrigenomic trial. Oxid Med Cell Longev. 2018;2018:5461436. doi:10.1155/2018/5461436

31. Snopek L, Mlcek J, Sochorova L, et al. Contribution of red wine consumption to human health protection. Molecules. 2018;23 (7):1684. doi:10.3390/molecules23071684

32. Boban M, Modun D, Music I, et al. Red wine induced modulation of vascular function: separating the role of polyphenols, ethanol, and urates. J Cardiovasc Pharmacol. 2006;47(5):695-701. doi:10.1097/ 01.fjc.0000211762.06271.ce

33. Modun D, Music I, Vukovic J, et al. The increase in human plasma antioxidant capacity after red wine consumption is due to both plasma urate and wine polyphenols. Atherosclerosis. 2008;197 (1):250-256. doi:10.1016/j.atherosclerosis.2007.04.002

34. Mudnic I, Budimir D, Modun D, et al. Antioxidant and vasodilatory effects of blackberry and grape wines. J Med Food. 2012;15(3):315321. doi:10.1089/jmf.2011.0129 


\section{Supplementary material}

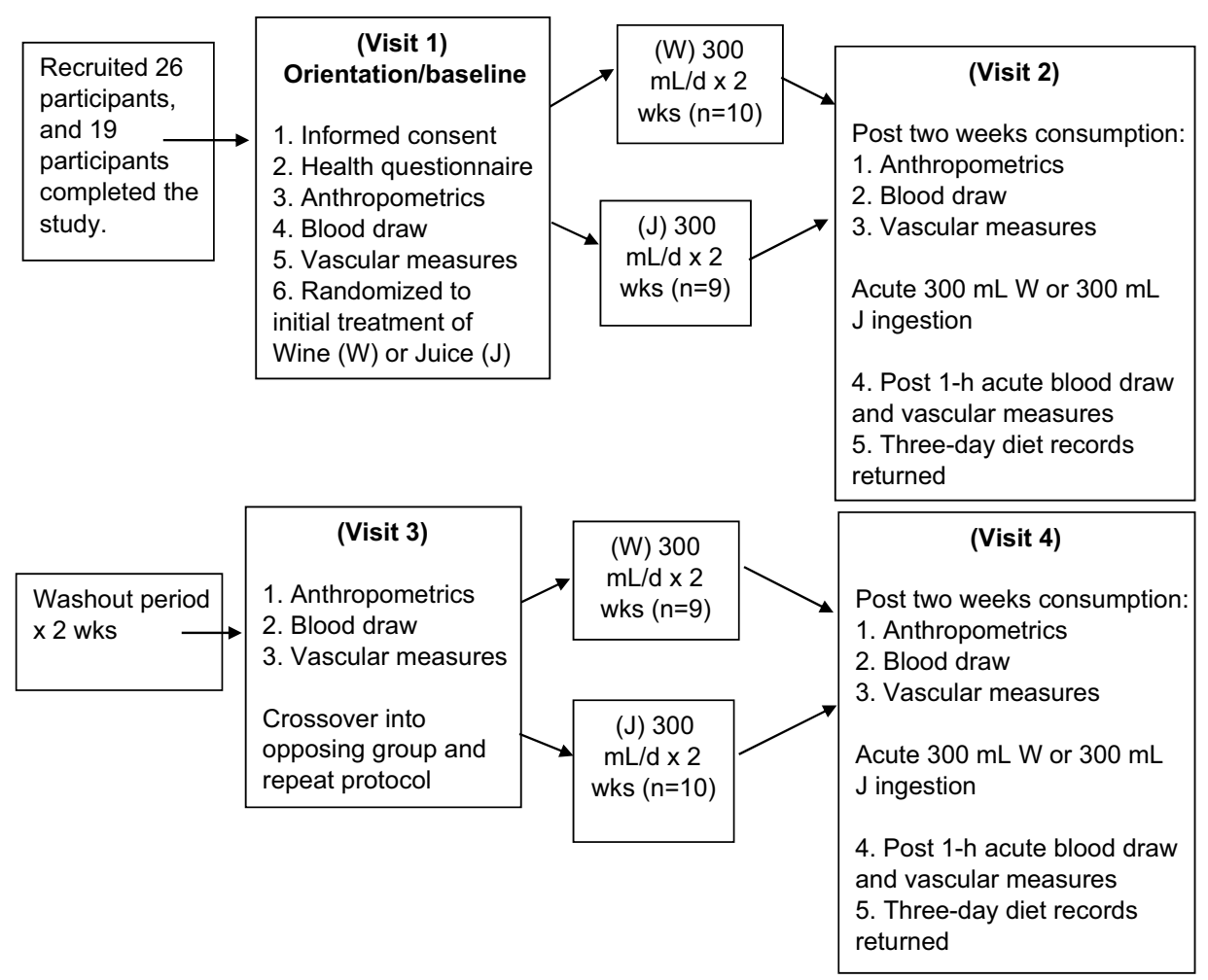

Figure SI Study Flow Diagram.

\section{Publish your work in this journal}

The International Journal of Wine Research is an international, peerreviewed open-access journal that focuses on all scientific aspects of wine, including: vine growing; wine elaboration; human interaction with wine; health aspects of wine. The journal provides an open access platform for the reporting of evidence based studies on these topics. The manuscript management system is completely online and includes a very quick and fair peer-review system, which is all easy to use. Visit http://www.dovepress.com/testimonials.php to read real quotes from published authors. 